\title{
Médiévales
}

Langues, Textes, Histoire

79 | automne 2020

Éthiopie, Nubie, Égypte

\section{Paul Fermon, Le Peintre et la carte. Origines et essor de la vue figurée entre Rhône et Alpes (XIV ${ }^{e-X V}$ siècle)}

Turnhout, Brepols («Terrarum orbis », 14), 2018, 507 p.

\section{Juliette Dumasy-Rabineau}

\section{(2) OpenEdition}

Journals

Édition électronique

URL : https://journals.openedition.org/medievales/11153

DOI : 10.4000/medievales. 11153

ISSN : 1777-5892

Éditeur

Presses universitaires de Vincennes

Édition imprimée

Date de publication : 28 décembre 2020

Pagination : 228-230

ISBN : 978-2-37924-146-8

ISSN : 0751-2708

Référence électronique

Juliette Dumasy-Rabineau, «Paul Fermon, Le Peintre et la carte. Origines et essor de la vue figurée entre Rhône et Alpes (xıve $x v^{e}$ siècle) », Médiévales [En ligne], 79 | automne 2020, mis en ligne le 28 janvier 2021, consulté le 07 janvier 2023. URL : http://journals.openedition.org/medievales/11153 ; DOI https://doi.org/10.4000/medievales.11753

Ce document a été généré automatiquement le 7 janvier 2023.

Tous droits réservés 


\title{
Paul Fermon, Le Peintre et la carte. Origines et essor de la vue figurée entre Rhône et Alpes (XIV $v^{e-X V}$ siècle)
}

Turnhout, Brepols (« Terrarum orbis », 14), 2018, 507 p.

\author{
Juliette Dumasy-Rabineau
}

\section{RÉFÉRENCE}

Paul Fermon, Le Peintre et la carte. Origines et essor de la vue figurée entre Rhône et Alpes (XIv ${ }^{e-X v}$ siècle), Turnhout, Brepols (« Terrarum orbis », 14), 2018, 507 p.

1 Ce livre, qui est la version publiée de la thèse de doctorat présentée par Paul Fermon à l'École Pratique des Hautes Études en 2017, a pour projet d'étudier «toutes les catégories de représentations d'espaces locaux qui furent élaborées entre la vallée du Rhône et les Alpes à la fin du Moyen Âge", dont il faut préciser qu'il s'agit de représentations figurées. L'auteur place ses travaux dans le sillage de ceux menés par Patrick Gautier Dalché (son directeur de thèse), dans une volonté affichée d'aborder la question des représentations de l'espace et de la cartographie sous un angle culturel. Il en tire une approche large du sujet, en étudiant tous les types de support pouvant receler des représentations de l'espace: cartes, plans, schémas, mais aussi sceaux, enluminures, fresques et peintures murales, retables. Cette approche, qui comprend dans une même problématique œuvres d'art et documents cartographiques, est l'un des intérêts majeurs de cette recherche.

2 Dans un premier temps, l'auteur examine la culture cartographique dans la vallée du Rhône (chap.1), en particulier à la cour des papes d'Avignon, où ont circulé dès les années 1320 les cartes de Marino Sanudo et de Paulin de Venise - ainsi que des cartes marines -, où Opicinus de Canistris a dessiné les siennes. Un siècle plus tard, la cour du roi René en Provence est aussi un foyer de production cartographique, qu'il s'agisse de mappemondes ou de représentations de terres faisant partie de ses titres et qu'il 
commande à ses peintres (cartes perdues de la Loire, de la Provence, vues de Rome et de Jérusalem). Ce développement d'une culture cartographique va de pair, selon l'auteur, avec l'essor de la représentation des lieux sous une forme naturaliste dans la production artistique. Ce phénomène est bien connu, et correspond à ce que les historiens de l'art ont appelé l'irruption de la nature ou du paysage dans l'art au tournant des $\mathrm{XIII}^{\mathrm{e}}$ et $\mathrm{XIV}^{\mathrm{e}}$ siècles. P. Fermon montre à quel degré de remarquable précision descriptive les peintres ont abouti dans les vues de monuments, villes et paysages qu'ils insèrent dans leurs œuvres. À travers son étude de la peinture provençale aux $\mathrm{XIV}^{\mathrm{e}}$ et $\mathrm{XV}^{\mathrm{e}}$ siècles (chap.2), il met en lumière de magnifiques œuvres (chapelle Saint-Martial du Palais des papes, Couronnement de la Vierge d'Enguerrand Quarton), qui ont pour point commun de figurer de façon fidèle des lieux et édifices connus des peintres et de leurs commanditaires - avec parfois quelques entorses à la réalité permises par le contexte de la commande artistique. Il montre aussi les recherches et innovations effectuées par les peintres: l'utilisation de la vue en perspective, construite selon un point de vue plus ou moins élevé (parfois fictif ou reconstitué) et un angle plus ou moins oblique (il conviendrait à cet égard de clarifier les notions de vue à vol d'oiseau et de perspective cavalière). Cette forme de dessin a aussi pour caractéristique de s'appuyer sur le "portrait » (terme fréquent dans les sources, et qui renvoie au dessin au trait) pour figurer les monuments. Or, ces portraits d'architecture (chap.3) peuvent être aussi repérés dans des contextes autres qu'artistiques, sur des sceaux, des registres administratifs ou des plans d'architecture. P. Fermon évoque ainsi ce plan (perdu) du palais des papes commandité en 1415 par l'empereur Sigismond, ou ce portrait du Mont Viso (lui aussi disparu), élaboré en 1475 en vue du percement d'un tunnel.

3 P. Fermon aborde ensuite un important corpus de plans et cartes qu'il classe en dossiers documentaires établis selon l'identité de leurs commanditaires ou destinataires, appelés généralement «figures » et "portraits » dans les sources de l'époque. Dans un premier temps, il s'intéresse à des figures établies dans ou pour le milieu ecclésiastique à l'occasion de litiges sur des droits seigneuriaux (chap.4). De façon remarquable, tous ces conflits portent sur des milieux humides ou aquatiques, mais les pièces prennent des formes différentes : plan des étangs de Mauguio pour le partage de droits de dîmes (milieu du $\mathrm{XIV}^{\mathrm{e}}$ siècle), schémas géométriques de jurisconsultes à propos de droits maritimes de l'abbaye de Lérins et dessin pour garder la mémoire d'un accord de propriété à Saint-Gilles (deuxième moitié $d u \mathrm{xv}^{\mathrm{e}}$ siècle). Selon l'auteur, ces trois cas montrent l'intérêt que portaient les milieux ecclésiastiques aux représentations figurées de l'espace, comme cela était déjà visible à Avignon. Un rôle important a aussi été joué par les officiers princiers, à l'origine de plusieurs cartes territoriales, c'est-àdire «mises en œuvre par le pouvoir royal ou princier» (chap.5). En Dauphiné, en Savoie ou en Provence, ces derniers ont eu recours à plusieurs reprises au $\mathrm{Xv}^{\mathrm{e}}$ siècle à des plans et cartes dans le cadre d'enquêtes sur des conflits frontaliers. Le dossier delphinal, dans les années 1420-1430 est particulièrement intéressant (figures de la vallée de Château-Dauphin, de Bellecombe...). De façon fort convaincante, P. Fermon voit dans ces récurrences l'initiative de Mathieu Thomassin, un officier dauphinois formé au droit à Orléans et qui connaissait probablement l'usage, alors bien établi au Parlement de Paris, de cartes pour résoudre les litiges ayant une dimension foncière ou territoriale. Comme le dit P. Fermon, le recours à des cartes est certainement à attribuer au modèle offert par le Parlement; que le Dauphiné ait été parmi les premières principautés à en user n'est pas un hasard. La Bourgogne suivra peu après. 
Les cartes de justice constituent justement le dernier dossier documentaire étudié (chap.6), dont la belle "figure accordée " du Rhône entre Avignon et Barbentane, réalisée par le célèbre peintre Nicolas Dipre en 1514. Elles sont un des aspects les plus remarquables du développement de la cartographie locale à partir du XIve siècle. Le dernier chapitre propose une réflexion synthétique sur les méthodes à l'œuvre dans les "vues figurées ", que P. Fermon définit finalement comme des documents ayant pour sujet principal la représentation d'espaces locaux sous forme d'une vue dessinée ressemblante, ce qui évacue de fait la plupart des œuvres d'art qui montrent les lieux et paysages sous forme d'un décor d'arrière-plan. On notera cependant qu'il ne faut pas exclure de la réflexion les plans et cartes qui ne sont pas construits sur la logique de la vue, ni le fait que nombre de vues figurées comportent des éléments schématiques. En outre, il ne semble pas très clair si la notion de vue figurée renvoie à un procédé formel ou à une catégorie de documents spécifique.

4 L'ouvrage est remarquable à plus d'un titre, par la nouveauté et l'originalité du sujet, par les belles trouvailles archivistiques de son auteur, par la précision de ses analyses de documents, qu'il s'agisse d'œuvres d'art ou de sources de la pratique, et l'on ne peut que saluer l'aisance avec laquelle il va des unes aux autres. Le propos est servi par un cahier d'illustrations abondant (plus d'une centaine de figures en couleur). L'apport de cette thèse est d'autant plus précieux qu'elle s'inscrit dans une série de travaux qui ont permis, depuis une dizaine d'années, un renouvellement important de la recherche sur les représentations de l'espace et la cartographie à l'échelle locale et régionale en France, comme l'a montré l'exposition qui s'est tenue aux Archives nationales à l'automne 2019 (et qui présentait plusieurs des cartes qu'a étudiées l'auteur) ${ }^{1}$. P. Fermon aurait eu d'ailleurs intérêt à utiliser davantage les fruits de cette recherche, par exemple en s'appuyant sur les études de Monique Pelletier, Camille Serchuk ou Raphaëlle Skupien, qui ont travaillé sur le même type de sources que lui. Néanmoins, l'étude permet d'établir un certain nombre de faits en tous points convaincants. La diffusion d'une culture cartographique précoce et multiforme parmi les élites lettrées, ainsi que le goût de ces mêmes cercles pour les décors incluant des représentations de l'espace, sont fort bien montrés, en particulier à la cour d'Avignon. Le rôle des milieux ecclésiastiques, princiers et judiciaires dans la production de cartes et plans locaux, et la nature litigieuse de celle-ci sont justement mis en avant. Les liens étroits entre art et cartographie se constatent enfin dans l'émergence parallèle d'œuvres d'art représentant des lieux identifiables et de cartes locales, la mise au point de procédés graphiques communs, et dans le fait que les peintres se font cartographes. C'est ce qui fait toute l'originalité de cette cartographie figurative, que le livre met à l'honneur de fort belle manière.

\section{NOTES}

1. "Quand les artistes dessinaient les cartes. Vues et figures de l'espace français, Moyen Âge et Renaissance », Musée des Archives Nationales, Hôtel Soubise, 25 septembre 2019 - 6 janvier 2020. 


\section{AUTEURS}

\section{JULIETTE DUMASY-RABINEAU}

Université d'Orléans, POLEN 